\title{
Novel anti-denatured collagen humanized antibody D93 inhibits angiogenesis and tumor growth: An extracellular matrix-based therapeutic approach
}

\author{
FLAVIA PERNASETTI ${ }^{1,3}$, JEFFREY NICKEL ${ }^{1}$, DEREK CLARK ${ }^{1}$, PATRICK A. BAEUERLE $^{1,2}$, \\ DENNIS VAN EPPS ${ }^{1}$ and BRUCE FREIMARK ${ }^{1}$ \\ ${ }^{1}$ Cell-Matrix, Inc., a subsidiary of Micromet Corporation, 2110 Rutherford Road, Carlsbad, \\ CA 92008, USA; ${ }^{2}$ Micromet AG, Staffelseestr. 2, D-81477 Munich, Germany
}

Received July 10, 2006; Accepted August 24, 2006

\begin{abstract}
Matrix metalloproteases (MMPs) secreted by both tumor and endothelial cells proteolytically degrade collagen during tumor growth and neo-vascularization. This exposes cryptic binding sites on collagen with functional relevance for angiogenesis. In this report, we characterized a novel humanized monoclonal IgG1 antibody, D93. After humanization, the antibody retained the binding specificity of the parental murine IgM antibody for denatured (dn) collagen. D93 recognized dn-collagen but not native (nat) collagen of different species, including mouse, chicken, and human, indicating that its cryptic binding site(s) is conserved across species. In immunohistochemistry (IHC) studies, D93 stained the basement membrane of blood vessels in several xenograft human tumors or in surgically removed tumor tissues from patients with different types of malignancies. D93 staining was rarely or not present in normal blood vessels of healthy tissues. In in vivo experiments, D93 significantly inhibited basic fibroblast growth factor (bFGF)-induced angiogenesis in chick embryo chorioallantoic membrane (CAM) and the tumor growth of pre-established orthotopic human breast (MDA-MB-435) tumors in mice. D93 i.v. administered in mice was subsequently detected in the subendothelial basement membrane of tumor blood vessels but not blood vessels of normal tissues. Inhibition of growth of pre-established orthotopic human breast MDA-MB-435 tumors was more effective when D93 was combined with Taxol, than either treatment alone. In addition, tumors from animals treated with D93 and/or Taxol showed significantly reduced levels of the endothelial cell-marker CD31. Our data suggest that blockade of cryptic epitopes exposed on collagen IV during angiogenesis and tumor growth by a monoclonal
\end{abstract}

Correspondence to: Dr Flavia Pernasetti, ${ }^{3}$ Present address: 8264 Calle Calzada, San Diego, CA 92126, USA

E-mail: fpernase@hotmail.com

Key words: antiangiogenic therapy, extracellular matrix, denatured collagen, monoclonal antibody antibody may provide a novel therapeutic modality for treatment of cancer and pathogenic neo-vascularization.

\section{Introduction}

The extracellular matrix (ECM) is a molecular network composed of two main compartments: the basement membrane and the interstitial matrix. The ECM was originally thought to function only as a mechanical support to cells and tissues but this concept has changed in the past years with findings that the ECM contains a wealth of biochemical information with importance for cellular processes including adhesion, migration, and proliferation (1). The perivascular ECM is particularly involved in angiogenesis, a process involving the formation of new blood vessels from preexisting vasculature occurring during physiologic and pathologic conditions, including tumor growth and metastasis (2-6).

Type IV collagen, a major component of the perivascular ECM, is secreted by both endothelial and tumor cells during angiogenesis, and accumulates in the subendothelial space $(7,8)$. Matrix metalloproteases secreted by both tumor and endothelial cells during angiogenesis have been shown to remodel or denature type IV collagen, and to expose cryptic sites on collagen that play a major role in inducing tumor and endothelial cell adhesion, migration, and proliferation $(9,10)$.

Using a subtractive immunization process, Xu et al have generated murine monoclonal antibodies that recognize cryptic epitopes on thermally dn-collagen (11). Two murine IgM monoclonal anti-dn-collagen antibodies have been developed: HUIV26, which preferentially binds to dn- and not to nat-collagen type IV, and HUI77, which preferentially binds to denatured and not to nat-collagen types I, II, III, IV, and $\mathrm{V}$ (11).

In this report, we show the anti-angiogenic and anti-tumor properties of the recombinant humanized anti-dn-collagen antibody D93, derived from the murine HUI77 antibody. D93 was shown to preferentially bind to human and mouse dn-collagen type IV, compared to nat-collagens or other ECM components. The humanized anti-dn-collagen antibody showed preferential binding to cryptic epitopes present on dn-collagen type IV within the basement membrane of blood 
vessels in human xenograft tumors from mice and tumor tissues from patients. Inhibition of angiogenesis by D93 was demonstrated using a basic FGF (bFGF) chick chorioallantoic membrane (CAM) model. D93 was also shown to significantly inhibit pre-established human breast orthotopic (MDA-MB435) xenograft tumor growth in a dose-dependent manner and to preferentially target tumor tissues when injected in vivo. Treatment with D93 also reduced the levels of endothelial cell marker CD31 in these tumors. Combination treatment of D93 with Taxol produced additive tumor inhibitory effects in this model. These findings suggest that blockade of cryptic epitopes exposed on collagen during angiogenesis using the anti-dn-collagen antibody D93 inhibits angiogenesis and tumor growth, and may be a novel ECM-based approach for the treatment of solid tumors.

\section{Materials and methods}

Reagents and chemicals. Purified collagens, fibronectin, laminin, and hydrocortisone were obtained from Sigma-Aldrich (St. Louis, MO). Rat polyclonal anti-mouse PECAM (CD31) antibody was obtained from BD Biosciences Pharmingen (San Diego, CA). Rabbit polyclonal anti-mouse collagen type IV antibody was purchased from Chemicon International (Temecula, CA). Mouse anti-human Factor VIII-related antigen was purchased from BioGenex (San Ramon, CA). Goat antimouse IgG Alexa Fluor 568, goat anti-rat IgG Alexa Fluor 568, goat anti-rabbit IgG Alexa Fluor 488, goat anti-human IgG Alexa Fluor 488 secondary antibodies, and StreptavidinConjugate Alexa Fluor 488 were obtained from Molecular Probes (Eugene, OR). OCT was obtained from VWR (San Francisco, CA). Mounting medium with DAPI (Vectashield ${ }^{\circledR}$ ) was purchased from Vector Laboratories (Burlingame, CA).

Cell lines and cultures. M21 human melanoma cells from Cell-Matrix, Inc. were grown in DMEM cell culture medium (HyClone, Logan, UT) supplemented with $10 \%$ fetal bovine serum (FBS; HyClone). BxPC-3 human pancreatic tumors were obtained from AntiCancer Inc. (San Diego, CA), MDA-MB-435 human breast tumor cells were obtained from the National Cancer Institute Cell Line Repository (Bethesda, MD) and MDA-MB-231 human breast tumor cells were obtained from ATCC (Manassus, VA). MDA-MB-435 and MDA-MB-231 cells were cultivated as monolayers in RPMI-1640 cell culture medium (Mediatech, Herndon, VA) supplemented with $10 \% \mathrm{FBS}$. All cells were cultured at $37^{\circ} \mathrm{C}$ in a humidified atmosphere of $5 \% \mathrm{CO}_{2}$.

Generation and production of D93 and DP28 antibodies. D93, a humanized IgG1 $\kappa$ anti-dn-collagen antibody, was generated based on heavy and light chain variable region sequences of the murine monoclonal IgM HUI77 antibody. The humanization process was performed by Applied Molecular Evolution Inc. (San Diego, CA), using an affinity maturation process. An isotype negative control antibody DP28 was generated by replacing the heavy chain variable domain of D93 with that of DP28. CDRs 1, 2, and 3 of the DP28 heavy chain differ in 16 amino acids from those of D93, leading to no dn- or nat-collagen binding by DP28. NS0 cells expressing D93 or DP28 antibody using the Glutamine Synthetase (GS) system (Lonza Group Ltd., Switzerland) were grown in Gibco Hybridoma-SFM media (Carlsbad, CA) supplemented with $5 \%$ dialyzed fetal calf serum. Cells were grown in stirred bioreactors and scaled up to 151 before harvesting of cell culture supernatants. Humanized IgG was purified using a two-step process starting with protein A affinity chromatography followed by ion exchange chromatography.

Solid phase ELISA. Ninety-six-well ELISA microtiter plates were coated ( $50 \mu \mathrm{l} /$ well) with ECM proteins $(10-25 \mu \mathrm{g} / \mathrm{ml}$ in PBS, Sigma, St. Louis, MO) for $18 \mathrm{~h}$ at $4^{\circ} \mathrm{C}$. All subsequent steps were performed at room temperature (RT). Thermal denaturation of collagen was achieved by boiling collagen for $15 \mathrm{~min}$ followed by cooling in ice for $10 \mathrm{~min}$. Plates were blocked with $50 \mu \mathrm{l} /$ well of $1 \%$ BSA in PBS (Mediatech, Herdon, VA) for $1 \mathrm{~h}$. D93 was diluted in 1\% BSA in PBS (50 $\mu \mathrm{l} /$ well) and incubated for $1 \mathrm{~h}$. The plates were washed three times with PBS, and incubated with peroxidaseconjugated goat anti-human antibody (Caltag Laboratories, Burlingame, CA) diluted 1:2000 in 1\% BSA in PBS for $1 \mathrm{~h}$. The plates were then washed three times with PBS and developed with o-phenylenediamine (OPD) substrate (Sigma) $(0.4 \mathrm{mg} / \mathrm{ml})$ in $80 \mathrm{mM}$ citrate phosphate-buffer $(\mathrm{pH} \mathrm{5.0)}$. The colorimetric reaction was stopped by addition of $12 \mu \mathrm{l} /$ well of $4 \mathrm{~N} \mathrm{H}_{2} \mathrm{SO}_{4}$. Reactivity was measured with an ELISA plate reader at a wavelength of $490 \mathrm{~nm}$. All samples were tested in duplicate.

Chick Chorioallantoic Membrane (CAM) assay. Ten-day old chick embryos were purchased from McIntyre Farms (Santee, $\mathrm{CA}$ ). The embryos were incubated at $37^{\circ} \mathrm{C}$ with ambient humidity. Preparation of CAM was performed as described by Brooks et al (12). In brief, the angiogenesis stimulator bovine bFGF (75 ng in $10 \mu 1$ of PBS; R\&D Systems, Minneapolis, $\mathrm{MN}$ ) or vehicle (PBS) was placed on a filter disk on the CAM. D93, DP28 control antibody, or PBS was added to the filter after 24 and $48 \mathrm{~h}(25 \mu \mathrm{g}$ antibody/egg/treatment). At the end of the three-day period, the region of the CAM covered by the filter disk was excised, inverted, and the number of blood vessel branches contained within the disk surface was determined in a blinded fashion by two independent observers, using a high-powered dissecting microscope. CAM assays were performed with a minimum of 10 embryos per condition tested.

\section{Immunohistochemistry}

D93 direct binding to tissue. Fresh tumor or CAM tissue was isolated from mice or chicken eggs, embedded in OCT compound, and snap-frozen in liquid nitrogen. Tumor sections $(5-\mu \mathrm{m})$ were allowed to air-dry and then fixed in cold acetone for $10 \mathrm{~min}$. All subsequent steps were performed at RT. Sections were blocked with $2.5 \%$ goat serum in PBS for $30 \mathrm{~min}$, followed by incubation with primary antibodies D93, DP28 $(50 \mu \mathrm{g} / \mathrm{ml})$ or anti-mouse collagen type IV (1:40), and co-stained with anti-CD31 (1:20), diluted in $2.5 \%$ goat serum in PBS for $45 \mathrm{~min}$. Tissue was then washed three times in PBS and incubated with fluorescent secondary antibodies: goat anti-rat IgG-568 and goat anti-human IgG-488 or goat anti-rabbit IgG-488 (diluted 1:200 in $2.5 \%$ goat serum in PBS). Tissues were then washed three times with PBS and 
mounted using $100 \mu \mathrm{l}$ of DAPI-containing mounting media. Photomicrographs were taken using x20 lenses with a Nikon E800 microscope connected to a CoolSnap ${ }^{\mathrm{TM}}$ camera and MetaMorph software.

D93 direct binding to patient tumor and adjacent normal tissue. Human tumors and adjacent normal tissues were obtained from the National Disease Research Interchange (NDRI; Philadelphia, PA) or Ardais Corporation (Cytomyx; Lexington, MA) with approval from their respective institutional review boards. Malignant tumors and adjacent normal tissues were obtained from human patients no later than $24 \mathrm{~h}$ post-biopsy or surgery, and in each case, the nature of tissues was confirmed by pathological analysis. Tissues were handled with universal precautions, in accordance with the OSHA Blood Borne Pathogen Standard 1910.1030. Fresh human tissue sections were allowed to air-dry and then fixed in cold acetone for $10 \mathrm{~min}$. All subsequent steps were performed at RT. Endogenous avidin and biotin were blocked using the avidin/biotin blocking solution (Vector Labs, Burlingame, CA) for $30 \mathrm{~min}$, followed by blocking with $2.5 \%$ goat serum for $20 \mathrm{~min}$. Slides were then incubated with primary antibodies: mouse anti-human Factor VIII-related antigen (1:20) and biotinylated D93 (NHS-PEO4-biotin kit; Pierce, Rockford, IL; $25 \mu \mathrm{g} / \mathrm{ml}$ ), diluted in $2.5 \%$ goat serum in PBS for $2 \mathrm{~h}$. Tissues were then washed three times in PBS and incubated with fluorescent secondary antibodies diluted 1:200 (goat anti-mouse IgG Alexa Fluor 568 and streptavidin-conjugate Alexa Fluor 488) for $1 \mathrm{~h}$. Tissues were then washed three times with PBS and mounted using DAPI-containing media. Photomicrographs were taken at magnification $\mathrm{x} 10$.

In vivo antibody tissue distribution post-injection. Fresh tissues (tumor, lung, heart, liver, kidney, and brain) isolated from mice were air-dried and then fixed in cold acetone for $10 \mathrm{~min}$. All subsequent steps were performed at RT. Sections were blocked with $2.5 \%$ goat serum in PBS for $30 \mathrm{~min}$ and then incubated with primary antibody [rat anti-CD31 (1:30), in $2.5 \%$ goat serum PBS], for $45 \mathrm{~min}$. Tissues were washed three times in PBS and then incubated for $1 \mathrm{~h}$ with secondary fluorescent antibodies diluted 1:200 in $2.5 \%$ goat serum in PBS [goat anti-human IgG (Alexa 488) and goat anti-rat IgG (Alexa 568)]. After incubation with secondary antibodies, tissues were washed three times with PBS and mounted using DAPI-containing mounting media. Photomicrographs were taken at magnification $\mathrm{x} 20$.

CD31 staining and quantification. MDA-MB-435 tumor tissue sections were air-dried and fixed in cold acetone for $10 \mathrm{~min}$. All subsequent procedures were performed at RT. Sections were blocked with 5\% goat serum in PBS for $30 \mathrm{~min}$ and then incubated for $1 \mathrm{~h}$ with rat anti-mouse CD31 antibody diluted (1:30) in 5\% goat serum in PBS. Tissues were washed three times in PBS and then incubated for $1 \mathrm{~h}$ with secondary antibody Alexa 568 goat anti-rat $\operatorname{IgG}$ (diluted 1:100 in 2.5\% goat serum in PBS). Tissues were then washed three times in PBS and mounted using DAPI-containing mounting media. Photomicrographs were taken at magnification x20. To assess microvessel density in the tumors, CD31 staining was quantified according to the protocol described by Hoshida et al (13).

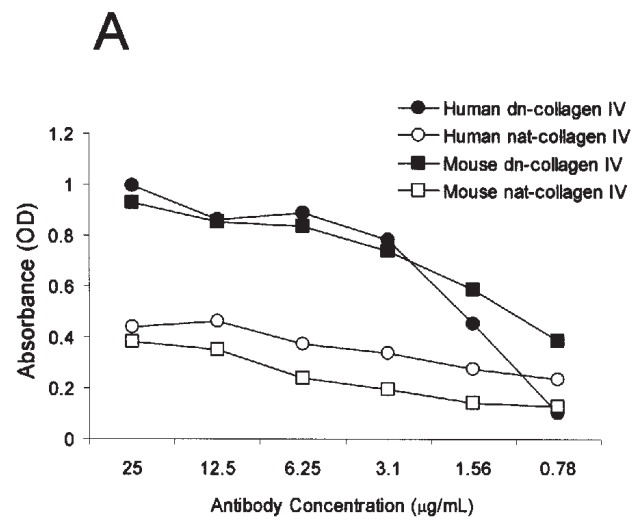

B

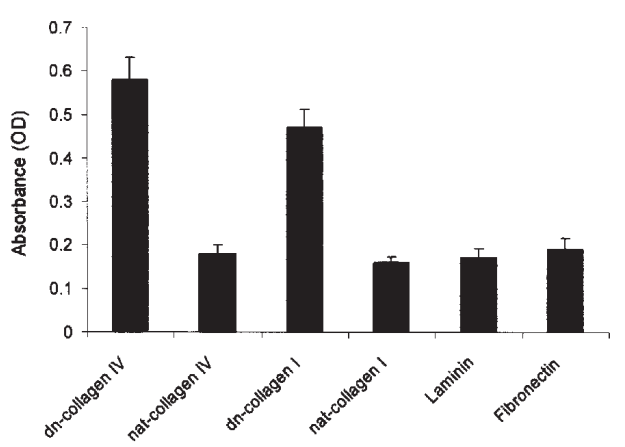

Figure 1. Binding specificity of D93. (A) Microtiter plates were coated with native and thermally denatured human or mouse type IV collagens $(10 \mu \mathrm{g} / \mathrm{ml})$. The humanized anti-dn-collagen D93 antibody was tested in serial dilutions by ELISA, as described in Materials and methods. (B) Assessment of D93 binding to other ECM proteins. Microtiter plates were coated with native and thermally denatured human type I and IV collagens, native laminin, and native fibronectin, all tested at $25 \mu \mathrm{g} / \mathrm{ml}$. D93 antibody was tested at $6.25 \mu \mathrm{g} / \mathrm{ml}$ and the ELISA was performed as described in Materials and methods.

Subcutaneous and orthotopic tumor models. All mouse studies were conducted in accordance with the regulations of the USDA Animal Welfare Act, in compliance with the Animal Welfare Assurance and under a protocol approved by the Institutional Animal Care and Use Committee at Perry Scientific, Inc. (San Diego, CA). Female NCR nu/nu (nude) mice (5- to 6-weeks old) were purchased from Simonsen Laboratories (Gilroy, CA). Animals were acclimated for at least 4 days and kept at four animals per cage. Prior to implantation of tumor cells, the animals were anesthetized and cells were implanted. MDA-MB-435 and MDA-MB-231 human breast tumor cells $\left(0.4 \times 10^{6}\right.$ and $2 \times 10^{6}$ cells, respectively, in $\left.50 \mu 1\right)$ were injected in the mammary fat pad of each animal. M21 human melanoma cells $\left(2 \times 10^{6}\right.$ cells in $\left.100 \mu \mathrm{l}\right)$ were implanted subcutaneously in the right flank of each animal. BxPC-3 1-2 $\mathrm{mm}^{3}$ tumor fragments from a stock tumor were implanted in the pancreas of each animal. At the end of the study, animals were sacrificed by cervical dislocation and tissues were collected and fresh frozen in OCT for immunohistochemistry analysis.

Statistical analysis. Statistical analyses of the data generated in these studies were performed using Student's t-test. All analyses were performed using One-way ANOVA, adjusting for multiple comparisons (JMP Statistical Discovery Software, 
A
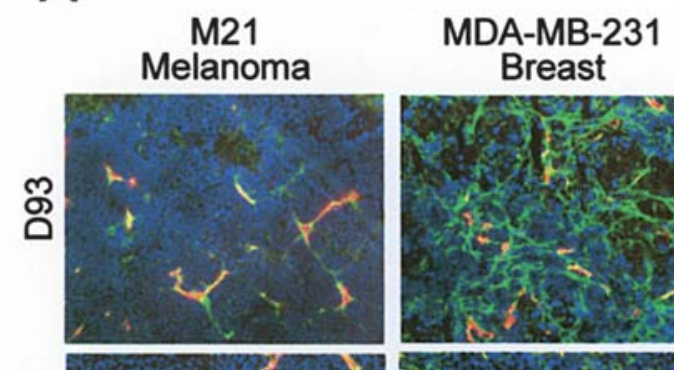
Breast
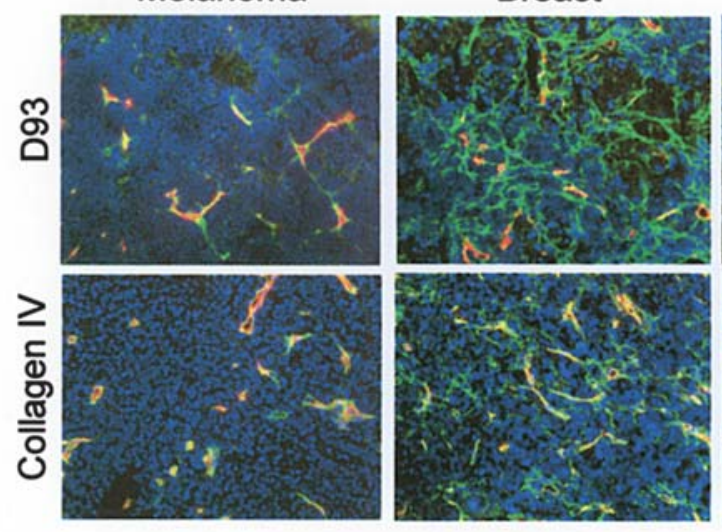

MDA-MB-435

Breast
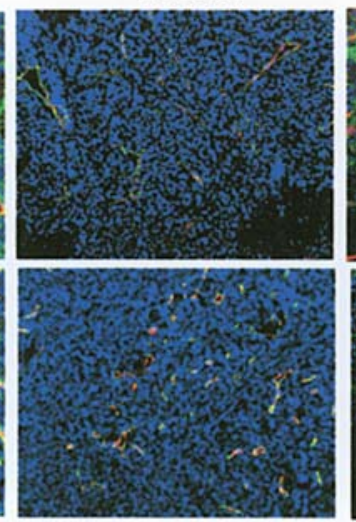

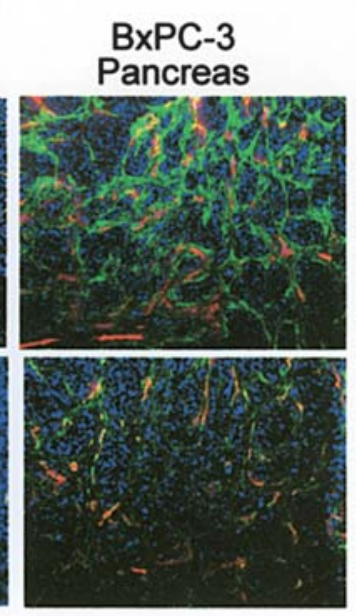

BxPC-3

B

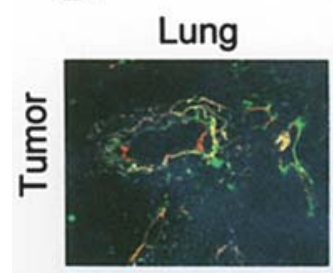

Kidney

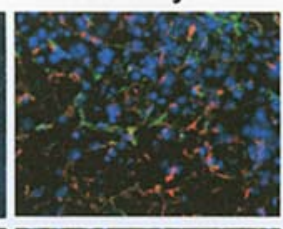

Colon

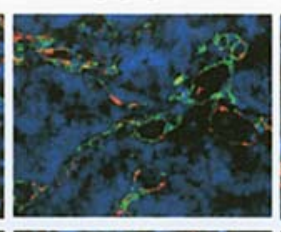

Pancreas
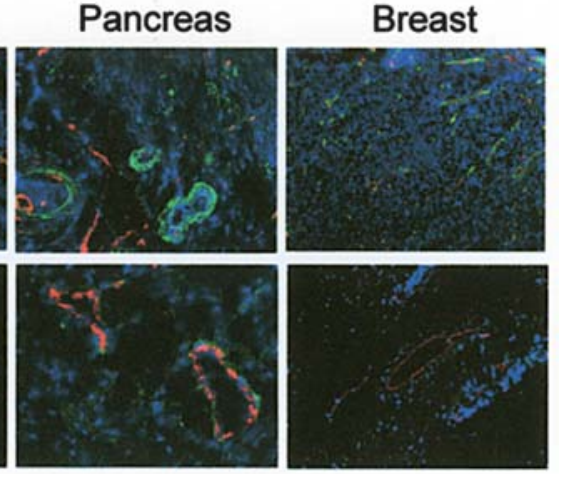

Figure 2. Binding of D93 to areas juxtaposed to blood vessels in tumor tissues. (A) Sections of xenogeneic human tumors (M21-melanoma, MDA-MB-231breast, MDA-MB-435-breast, and BxPC-3-pancreas) grown in mice were stained separately with D93 or anti-collagen IV antibodies. D93 was detected using goat anti-human IgG Alexa 488 antibody (green) and anti-collagen IV antibody was detected using anti-mouse IgG Alexa 488 antibody (green). All sections were co-stained with the endothelial cell marker anti-CD31 antibody, detected using goat anti-rat IgG Alexa 568 antibody (red). The nuclei were stained with DAPI (blue). Yellow shows overlap between green and red. Representative pictures of each tumor type are shown (magnification x20). (B) Human tumor and adjacent (Adj.) normal frozen tissue sections were obtained from commercial tissue banks. Tissues from patients were stained with biotinylated D93 anti-dn-collagen antibody and this antibody was detected using a Streptavidin-Alexa 488 (green). Sections were co-stained with the blood vessel marker anti-Factor-VIIIrelated antigen which was detected using goat anti-mouse IgG Alexa 568 antibody (red). The nuclei were stained with DAPI (blue). Yellow shows overlapping between green and red. Representative pictures of each tumor type and their respective adjacent normal tissue are shown (magnification x10).

v.5.1.1, Cary, NC). P-values $<0.05$ were considered statistically significant.

\section{Results}

D93 selectively binds to cryptic sites on dn-collagen IV. D93

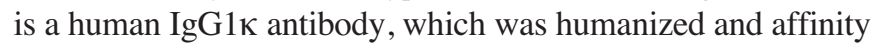
matured based on the CDR sequences of the murine HUI77 IgM antibody $(10,11)$. To evaluate whether D93 retained the ability to bind to dn-collagen after the humanization process, solid-phase ELISA binding studies were performed. As shown in Fig. 1A, D93 preferentially bound to dn- as compared to nat-mouse or human collagen type IV, and this binding was antibody dose-dependent. These observations indicate that antigen-recognition was retained in the course of the antibody humanization and affinity maturation process. Moreover, the ability of D93 to bind to dn-collagen type IV from both human and mouse indicates that this binding site(s) is conserved across species.
D93 preferentially binds to dn-collagen relative to other ECM components. To evaluate the binding of D93 to distinct ECM proteins, solid-phase ELISAs were performed using human laminin, fibronectin, and dn- and nat-collagens type I and IV. The humanized antibody D93 preferentially bound to dn-collagen compared to nat-collagen types I or IV or to other ECM components such as laminin and fibronectin, further demonstrating the antibody specificity for dn-collagen (Fig. 1B). The binding of D93 to dn-collagen types I and IV was expected, since D93 was derived from HUI77, which was previously shown to bind to dn-collagen types I through $\mathrm{V}$ (11).

D93 binds to sub-endothelial basement membrane of angiogenic blood vessels in tumors from various xenografts and from human patients. To determine whether the D93 antibody binding site in dn-collagen becomes accessible to the antibody in vivo, during invasive cellular processes such as angiogenesis and tumor growth, immunohistochemical (IHC) analyses 
Table I. Human tissue specimens analyzed by IHC.

\begin{tabular}{lll}
\hline Age (years) & Gender & \multicolumn{1}{c}{ Diagnostic } \\
\hline 65 & Male & Non-small cell lung carcinoma \\
61 & Male & Renal cell carcinoma \\
42 & Female & Colon adenocarcinoma \\
64 & Male & Pancreas adenocarcinoma (ductal) \\
68 & Female & Breast carcinoma (ductal) \\
\hline
\end{tabular}

were performed using various human xenograft tumor tissues grown in mice or various human patient primary tumors and adjacent normal tissues obtained post-surgery. Fig. 2A shows staining of various human xenograft tumors derived from M21 melanoma, MDA-MB-231 breast, MD-MBA-435 breast, and $\mathrm{BxPC}-3$ pancreas cell lines, with $\mathrm{D} 93$ or with a polyclonal anti-mouse collagen type IV antibody. Anti-CD31 antibody (PECAM) was used to identify blood vessels, and the nuclei were stained with DAPI. D93 exhibited selective binding to areas adjacent to blood vessels in all four xenografts tested, as detected by staining with an anti-CD31 antibody. Staining with the polyclonal anti-collagen IV antibody that recognizes both dn- and nat-collagen type IV showed a pattern similar to that of D93 in the tumor tissues, indicating that collagen type IV is predominantly present in the basement membrane of blood vessels in the xenograft tumor tissues. The similarity in staining patterns indicates that dn-collagen IV molecules are associated with newly formed vessels in all four human xenografts.

To determine the specificity of D93 binding in human tissues, sets of human patient tumors and adjacent normal tissues obtained post-surgery were stained with biotinylated D93 and with an antibody binding to Factor VIII (blood vessel marker). IHC evaluation of surgically removed tissues from human patients with colon, kidney, pancreas, breast and lung solid tumors (Table I) demonstrated that D93 antibody preferentially bound to areas juxtaposed to the tumor vasculature, with only minimal or absent D93 staining in adjacent normal tissues. These observations further support the preferential binding of D93 to dn-collagen type IV at sites of ECM remodeling and angiogenesis in human tumor tissue.

D93 inhibits angiogenesis. The ability of D93 to inhibit angiogenesis in vivo was evaluated using the bFGF CAM model. As shown in Fig. 3A, D93 significantly inhibited bFGFinduced blood vessel formation at both antibody concentrations tested (50 and $25 \mu \mathrm{g} /$ dose). D93 inhibited formation of new blood vessel branches by 70 and $53 \%$ compared to the bFGF + PBS control $(\mathrm{p}<0.05)$, and 66 and $47 \%$ compared to the $\mathrm{bFGF}+\mathrm{DP} 28$ group $(\mathrm{p}<0.05)$, respectively. No significant difference was observed between the bFGF + DP28 antibody and the bFGF + PBS treatments, as expected. There was a trend to greater inhibition of blood vessel formation with the highest concentration of D93 antibody tested (50 $\mu \mathrm{g} / \mathrm{dose})$ compared to the lower dose $(25 \mu \mathrm{g} / \mathrm{dose})$, however these differences did not reach statistical significance. Inhibition of blood vessel branching by D93 is shown in a photomicrograph in Fig. 3B. IHC analysis showed that D93 bound to the basement membrane of the developing blood vessels present in CAM tissue from chicken eggs treated with bFGF, while the control isotype antibody DP28 did not show specific binding (Fig. 3C). These observations suggest that the D93 binding site(s) is newly exposed in the developing blood vessels present in chicken CAM in response to bFGF treatment and that its blockade by the antibody can effectively suppress angiogenesis. These data demonstrate reactivity of D93 with chicken dn-collagen, confirming reactivity of D93 with dn-collagen across species.

Systemic administration of D93 inhibits orthotopic breast tumor growth in a dose-dependent fashion. The growth of solid tumors beyond 1-2 $\mathrm{mm}^{3}$ has been shown to depend on the development of new blood vessels or angiogenesis $(14,15)$. To characterize the effect of D93 on the growth of established orthotopic human breast (MDA-MB-435) xenograft tumors, mice were treated intravenously (i.v.) twice per week (b.i.w.) for 70 days, with the humanized anti-dn-collagen antibody D93. Treatment was initiated once the mean tumor volume reached $\sim 60 \mathrm{~mm}^{3}$. D93 was tested at three different doses: 100,10 , and $1 \mu \mathrm{g} /$ dose and control antibody DP28 was administered at $100 \mu \mathrm{g} /$ dose. The vehicle control group received $100 \mu 1$ of PBS per dose. The isotype control antibody DP28 had no effect on tumor growth compared to vehicletreated (PBS) animals, as shown in Fig. 4A. D93 at $100 \mu \mathrm{g} /$ dose (D93-100) significantly inhibited tumor growth compared to isotype control DP28 on Day 55 (43\%, p=0.048), Day 62 $(49 \%, \mathrm{p}=0.018)$ and Day $70(43 \%, \mathrm{p}=0.047)$. Tumor growth in the D93 $10 \mu \mathrm{g} / \mathrm{dose}$ (D93-10) group was not significantly different than DP28 control at any point in time, inhibiting tumor growth by $22 \%$ at Day 70 . D93 at $1 \mu \mathrm{g}$ /dose (D93-1) showed no effect on tumor growth compared to control groups at any point in time, when compared to the two control groups. These results show that bi-weekly treatment with $100 \mu \mathrm{g}$ D93 can significantly inhibit growth of preestablished human breast tumors growing orthotopically in mice. This effect was significant, dose-dependent, and specific, as it was not observed with the isotype control antibody or vehicle.

D93 in vivo tumor-targeting. The distribution and localization of i.v. administered D93 and isotype control antibody DP28 was determined by IHC analysis of tissues from mice bearing orthotopic MDA-MB-435 human breast xenograft tumors. Antibody distribution was assessed in tumor, kidney, liver, heart, lung, and brain tissues. D93 preferentially targeted areas juxtaposed to blood vessels in tumor tissue (Fig. 4B) and was rarely or not detected in normal tissues (brain, heart, lung, or liver), with the exception of the glomeruli of the kidneys. The isotype control antibody DP28 was not detectable in tumor tissue or in any of the normal tissues analyzed, except in the glomeruli of the kidneys. These results demonstrate that D93 injected in vivo preferentially targets to areas of new blood vessel formation present in the tumor xenograft tissue but not to established blood vessels present in healthy organs such as brain, heart, kidney (except glomeruli), lung, or liver. The presence of both D93 and the isotype control antibody in the glomeruli of the kidneys appears not specific 
A

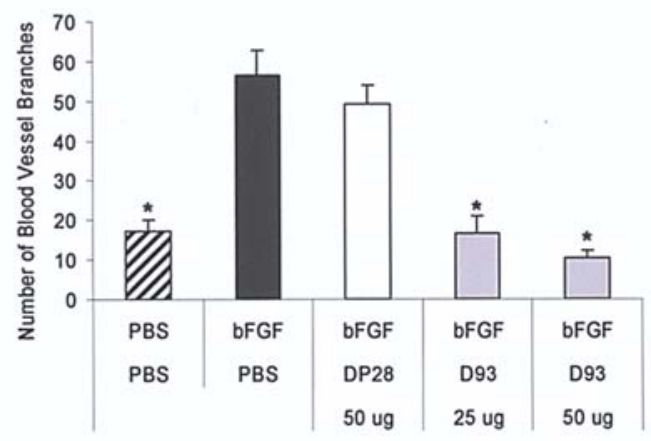

B

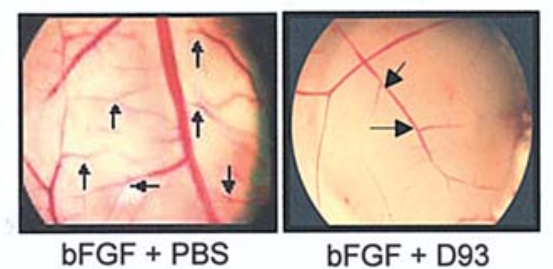

C

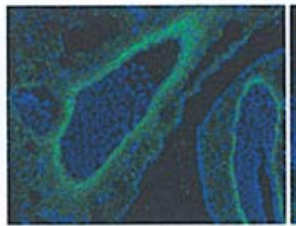

D93

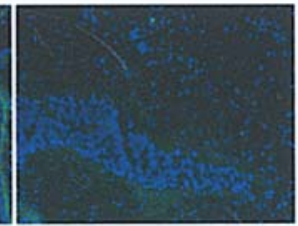

DP28
Figure 3. Anti-angiogenic effect of D93 in the CAM model. (A) bFGF (75 ng) was applied to a paper filter placed on the CAM, followed by antibody topical treatment 24- and 48-h after bFGF treatment. A control group that did not receive bFGF stimulation [diluent (PBS) only] was used as baseline. D93 was tested at two different concentrations ( 25 and $50 \mu \mathrm{g} /$ dose $)$, and the negative control isotype antibody DP28 was tested at $50 \mu \mathrm{g} / \mathrm{ml}$. At least ten eggs per group were evaluated in three independent experiments. Data are presented as mean blood vessel branches \pm SEM per treatment group. ${ }^{*}$ Statistically different from the $\mathrm{bFGF}+\mathrm{PBS}$ group and the $\mathrm{bFGF}+\mathrm{DP} 28$ control antibody group $(\mathrm{p}<0.05)$. (B) Representative images of CAM treated with bFGF + PBS (positive control) or with bFGF + D93 (50 $\mu \mathrm{g} /$ dose). (C) Representative images of D93 or control antibody $(50 \mu \mathrm{g} / \mathrm{ml})$ staining (green) on bFGFtreated CAM tissue. The nuclei were stained with DAPI (blue).

to D93, and may at least in part, be attributable to clearance of IgG antibodies and their complexes by the kidneys (16).

Combination with Taxol enhances the inhibitory effect of D93 on orthotopic breast tumor growth. Taxol ${ }^{\circledR}$ is a highly effective anti-neoplastic chemotherapeutic used for the treatment of advanced metastatic breast cancer (17). Recent reports showed that Taxol improves therapeutic efficacy in combination with anti-angiogenic agents (Miller KD, American Society of Clinical Oncology Annual Meeting, late breaking session, 2005). We treated mice bearing established orthotopic human breast MDA-MB-435 xenograft solid tumors $\left(60 \mathrm{~mm}^{3}\right)$ biweekly with the D93 antibody (100 $\mu \mathrm{g} /$ dose) as a single therapy or in combination with Taxol. Taxol was administered intraperitoneally (i.p.), once per week (q.w.) at $120 \mu \mathrm{g} / \mathrm{dose}$

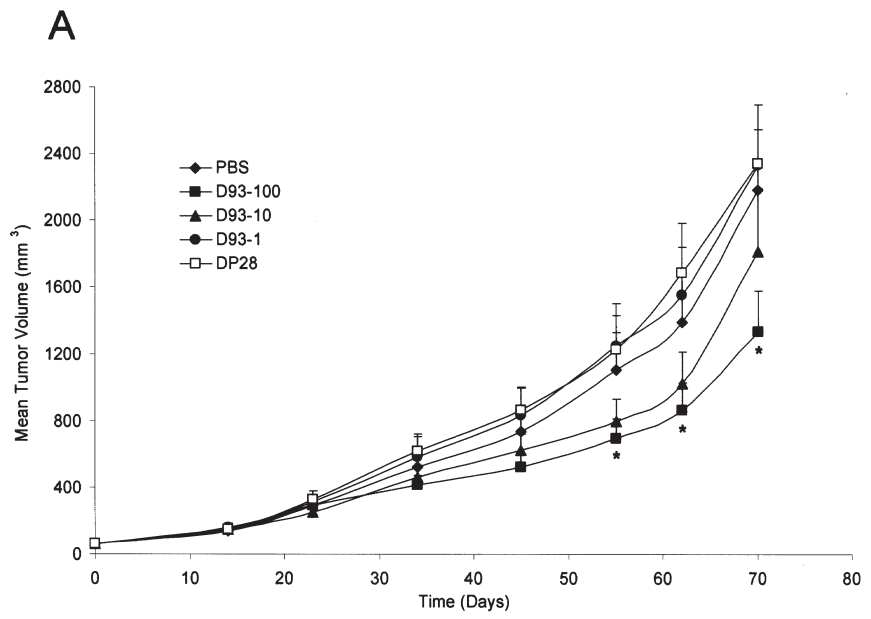

B
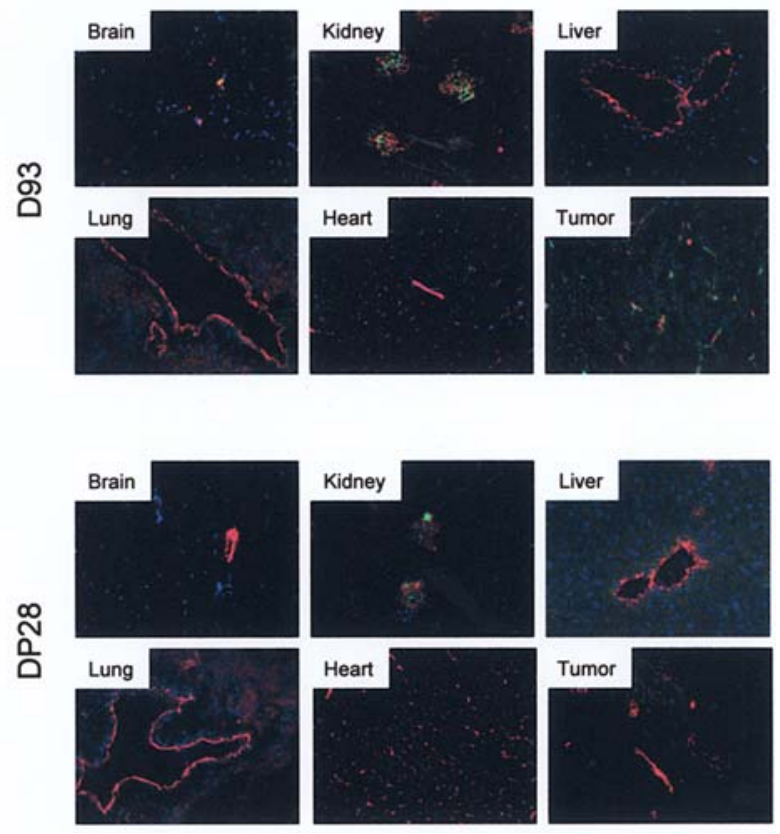

Figure 4. Anti-tumor activity of D93 in a pre-established orthotopic MDAMB-435 human breast model in mice. (A) MDA-MB-435 cells $\left(0.4 \times 10^{6}\right.$ cells/ animal) were implanted orthotopically in the mammary fat pad of female nude mice. Tumors were allowed to develop until they reached $60 \mathrm{~mm}^{3}$ volume, when mice ( $n=10$ animals/group) were randomized in four groups, to receive D93 at three concentrations $(100,10$ or $1 \mu \mathrm{g} / \mathrm{dose})$ or isogenic negative control antibody DP28 (100 $\mu \mathrm{g} /$ dose $)$. Treatments were performed i.v. twice per week for 70 days. Tumors were measured with the aid of an electronic caliper and tumor volume (V) was estimated using the formula $\mathrm{V}=\left(\mathrm{L} \times \mathrm{W}^{2}\right) / 2$, where $\mathrm{L}$ is the length and $\mathrm{W}$ is the width. Mean tumor volumes per treatment group \pm SEM were calculated and plotted as a function of time. "Statistically different than the PBS and DP28 control groups $(\mathrm{p}<0.05)$. (B) Distribution of D93 post-injection in mice. Tissues collected from mice treated with D93 and DP28 at $100 \mu \mathrm{g} /$ dose were obtained at the end of the study, fresh frozen in OCT, and sectioned for immunohistochemical analysis. Representative images of brain, kidney, liver, lung, heart, and tumor tissue are shown. Tissues were stained with goat anti-human IgG Alexa 488 to detect D93 (green). Sections were co-stained with the blood vessel marker CD31, detected with goat anti-rat IgG Alexa 568 (red). The nuclei were stained with DAPI (blue). Yellow shows overlapping between green (D93) and red (CD31) stainings.

( $\sim 6 \mathrm{mg} / \mathrm{kg}$, Tax-6) or $200 \mu \mathrm{g} / \mathrm{dose}(\sim 10 \mathrm{mg} / \mathrm{kg}$, Tax-10). The vehicle control group received $100 \mu 1 /$ dose of PBS (i.v. b.i.w.) and $100 \mu \mathrm{l} /$ dose of HBSS (i.p. q.w.). The isotype control group received DP28 antibody at $100 \mu \mathrm{g} /$ dose (i.v. b.i.w.). 
A

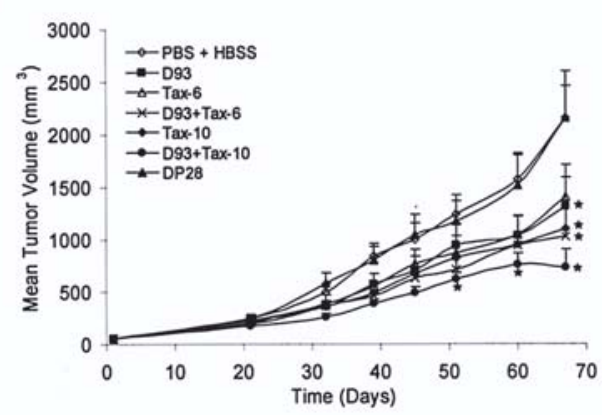

B

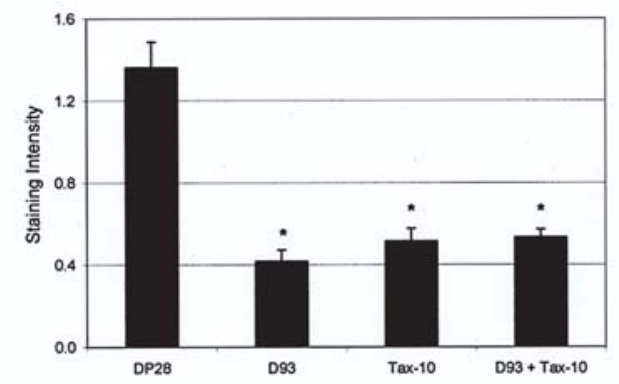

C

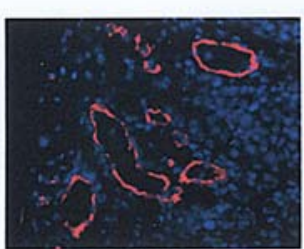

DP28

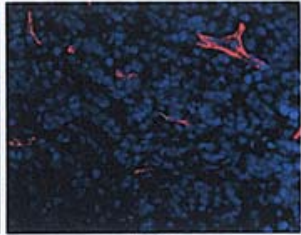

D93

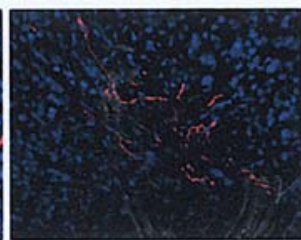

Tax-10

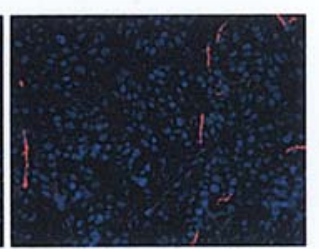

D93 + Tax-10

Figure 5. Anti-tumor activity of D93 in combination with Taxol. (A) D93, Taxol, and D93 + Taxol inhibit staged orthotopic MDA-MB-435 human breast tumor growth in mice. MDA-MB-435 cells $\left(0.4 \times 10^{6}\right.$ cells/animal) were implanted orthotopically in the mammary fat pad of female nude mice. Tumors were allowed to develop until they reached a mean $60 \mathrm{~mm}^{3}$, when mice were randomized into seven groups ( $\mathrm{n}=13$ animals/group). D93 or the isogenic negative control antibody DP28 (100 $\mu \mathrm{g} / \mathrm{dose})$ were administered i.v. twice per week. Taxol was given i.p. once per week at two different concentrations (6 or $10 \mathrm{mg} / \mathrm{kg})$ alone or in combination with D93. Treatments were carried out for 67 days. Tumors were measured with the aid of an electronic caliper and tumor volume (V) was estimated using the formula $\mathrm{V}=\left(\mathrm{L} \mathrm{x} \mathrm{W}^{2}\right) / 2$, where $\mathrm{L}$ is the length and $\mathrm{W}$ is the width. Mean tumor volumes per treatment group $\pm \mathrm{SEM}$ were calculated and plotted as a function of time. *Statistically different compared to vehicle control PBS + HBSS and DP28 control groups (p<0.05). (B) D93 treatment inhibits CD31 expression in tumors. Five tumors per treatment group were analyzed by immunohisto-chemistry. A minimum of 25 fields of view was measured per treatment group. The mean percent CD31 staining \pm SEM per area analyzed is shown for each treatment group. *Statistically significant differences relative to the isogenic DP28 antibody control group (p<0.05). (C) Tissues collected from mice treated with DP28, D93, Taxol 10 mg/kg (Tax-10) or D93 + Tax-10 were obtained at the end of the study, fresh frozen in OCT, and sectioned for immunohistochemical analysis. MDA-MB-435 tumor sections were stained with the blood vessel marker anti-CD31 antibody which was then detected using a goat anti-rat IgG Alexa 568 antibody (red). The nuclei were stained with DAPI (blue). Representative images (magnification x200) of CD31 staining for each treatment group are shown.

A decrease in tumor volume was seen in all treatment groups relative to the control groups (Fig. 5A), with the greatest therapeutic effect observed when animals were treated with D93 + Tax-10, with 66\% inhibition $(\mathrm{p}=0.0007)$ at the end of the study, Day 67. Treatment with D93 or Tax10 as single therapies also resulted in significant inhibition of tumor growth at the end of the study (39\%; $\mathrm{p}=0.04$ and 49\%; $\mathrm{p}=0.01$, respectively), when compared to controls. Monotherapy treatment with a lower dose of Taxol (Tax-6) showed a trend toward tumor inhibition $(35 \%, \mathrm{p}=0.068)$ but this did not reach statistical significance. However, the combination of D93 + Tax-6 significantly inhibited tumor growth (52\%, $\mathrm{p}=0.006$ ). No significant differences were observed between isotype control DP28 and PBS + HBSS control treatments, as expected. These data demonstrate that D93 can be functionally combined with Taxol and that this combination can achieve higher anti-tumor activity than either treatment alone.

D93 treatment reduces expression of CD31 in tumors. To better understand the mode of action of D93, tumors treated with D93, Taxol, or a combination thereof were analyzed by IHC for expression of the endothelial cell adhesion molecule CD31 (Fig. 5B and C). The expression of CD31 in MDA-MB-435 tumors was significantly reduced in treatment groups receiving D93, Tax-10, or D93 + Tax-10 when compared with the isotype control antibody DP28. Reduction of CD31 expression was 69,62 and $61 \%(\mathrm{p}<0.0001)$, respectively (Fig. 5B). These data suggest that treatment with D93 and Taxol, as single agents or in combination, significantly reduces tumor vessel density and expression of CD31 by tumor endothelial cells. 


\section{Discussion}

Historically, the ECM was thought to function mainly as a mechanical support for cells and tissues. However, recent studies have shown that the ECM contains biochemical information that plays an important role in the regulation of cellular activities (7), angiogenesis (8), the growth of primary tumors and metastases $(14,18)$. Numerous molecules have been shown to be involved in the regulation of angiogenesis and been considered as cancer therapeutic targets (19), but the majority of these studies and approaches have focused on growth factors and their receptors, cell adhesion molecules, and matrix-degrading proteases $(9,20-22)$. In comparison, relatively little emphasis has been placed on the ECM itself as a potential therapeutic target for control of neo-vascularization.

In this report, we demonstrate that targeting the ECM by a novel monoclonal human IgG1 antibody with specificity for dn-collagen is suitable to inhibit both tumor growth and angiogenesis, and is compatible with standard chemotherapy treatment. Previous work has demonstrated that murine IgM anti-dn-collagen antibodies can exert anti-angiogenic activity in vivo $(10,11)$, but such large and immunogenic antibodies are very difficult to develop for human treatment. The generation of the anti-dn-collagen D93 antibody, a human IgG1 antibody less likely to cause immunogenicity in humans, was achieved by humanization and affinity maturation of the variable domains of the HUI77 murine IgM antibody. D93 binding specificity for dn-collagen was retained during the humanization process, and the antibody was shown to bind to epitopes conserved across diverse species, a characteristic that may facilitate toxicological testing of the antibody in several models.

D93 appears to bind to a cryptic site(s) that is preferentially exposed within the subendothelial basement membrane of angiogenic and tumor-associated blood vessels, but not within the basement membrane of normal vessels. The binding of D93 antibody to areas of vascularization in human tumors of various types i.e. colon, breast, kidney, lung, and pancreas described in this report suggests that the cryptic binding site(s) recognized by this antibody are exposed in a variety of human solid tumors. Multiple types of tumors may thus share similar processes of angiogenesis and tumor growth that involve remodeling of collagen. Collagen is a ubiquitous component of the ECM present in all tissues, while the distinctive exposure of binding sites for D93 during angiogenesis may be the result of the secretion of MMPs or other proteases by tumors, stromal, and/or endothelial cells specifically at sites of tumor growth and neo-vascularization. The highly restricted occurrence of D93 immunoreactivity at tumor sites is in agreement with the hypothesis that dncollagen is largely present in areas of neo-vascularization, but not in existing vessels present in healthy tissues, where slow turnover of collagen occurs.

Previous studies indicate that cellular interactions with proteolyzed forms of ECM molecules such as collagen I, osteopontin, and laminin result in increased cell migration (23-25). Moreover, a murine anti-dn-collagen antibody HUIV26 was shown to inhibit angiogenesis and tumor growth in the chick CAM model (10). Thus, proteolytic remodeling of ECM proteins resulting in the exposure of cryptic sites on these molecules appears to play an important role in signaling through cell-surface receptors and/or adhesion molecules during angiogenesis. This process is a critical source of new regulatory signals during angiogenesis which may contribute to increased cell migration, adhesion, and proliferation. Consistent with this hypothesis, here we provide evidence that blockade of cell-ECM signaling by the anti-dncollagen antibody D93 results in inhibition of angiogenesis and tumor growth. Notably, angiogenesis induced by the cytokine bFGF was inhibited by D93 in a chick embryo CAM assay, and the expression of the endothelial cell marker CD31 was also significantly decreased in MDA-MB-435 tumors of animals treated with D93, to levels similar to those of animals treated with Taxol. This suggests that one mode of action of D93 is based on the reduction of endothelial cell density as reflected by decreased expression of CD31 in tumors.

Systemically administered D93 localized to areas juxtaposed to blood vessels in the MDA-MB-435 tumors, but not to blood vessels present in healthy tissues. These findings support a possible use of D93 or other anti-dn-collagen antibodies as markers of angiogenesis. If conjugated to imaging reagents, cytotoxic compounds, or radioactive isotopes, D93 could potentially be employed in novel diagnostics and/or in therapeutic uses.

In conclusion, the data presented here demonstrate that systemic administration of the humanized antibody D93 led to significant and dose-dependent inhibition of preestablished human breast MDA-MB-435 orthotopic tumors. The level of anti-tumor efficacy of D93 was consistent with an anti-angiogenic mode of action controlling tumor growth by constantly suppressing neo-vascularization. A similar level of activity was observed with other anti-angiogenic antibodies and small molecule inhibitors when given as a monotherapy (26).

Several recent studies have indicated that anti-angiogenic therapies can potentiate the effects of cancer treatments based on radiation or chemotherapy (27). The premise behind the design of novel combination therapies is therefore based on combining agents that act through different mechanisms. In this report, we have demonstrated that treatment of preestablished orthotopic human breast MDA-MB-435 tumors with D93 in combination with Taxol was more effective than either treatment alone. It is believed that D93 suppresses angiogenesis and tumor growth by blocking the signaling pathways that otherwise stimulate endothelial and/or tumor cell growth through interactions with the ECM. Taxol inhibits tumor growth by stabilizing polymerized tubulin, which leads to blockade of cell-cycle and induction of programmed cell-death $(28,29)$. These distinct modes of action may explain the additive effect of D93 and Taxol and support their use in combination treatment.

Targeting cryptic sites exposed on dn-collagen may have a number of advantages over other existing anti-angiogenic and anti-tumor therapeutic approaches. These include: i) target specificity, due to preferential binding to targets present mainly in areas of angiogenesis; ii) target stability, since collagen, which is produced by both host and tumor cells, is a structural molecule unlikely to mutate; iii) broad spectrum activity against a target exposed in various tumor types; and iv) low probability of acquired drug resistance based on the non- 
cellular nature of the target. Therefore, targeting matriximmobilized cryptic sites within ECM molecules using the anti-dn-collagen antibody D93 may be a highly specific, safe, and powerful approach for the treatment of neoplastic diseases in humans. Furthermore, because of its unique targeted mechanism of action, D93 could potentially be used in combination with other therapeutic agents acting through distinct mechanisms, resulting in a more effective treatment of solid tumors.

\section{Acknowledgements}

The authors would like to thank E. Pinney for technical assistance in the CAM assay.

\section{References}

1. Akalu A and Brooks P: Matrix, extracellular and interstitial. In Encyclopedia of Molecular Cell Biology and Molecular Medicine. 2nd edition. Meyers RA (ed). Wiley-VCH Verlag GmbH \& Co. KGaA, Weinheim, pp45-70, 2005.

2. Paku S and Paweletz N: First steps of tumor-related angiogenesis. Lab Invest 65: 334-346, 1991.

3. Gagne P, Akalu A and Brooks PC: Challenges facing antiangiogenic therapy for cancer: impact of the tumor extracellular environment. Expert Rev Anticancer Ther 4: 129-140, 2004.

4. Moses MA, Klagsbrun M and Shing Y: The role of growth factors in vascular cell development and differentiation. Int Rev Cytol 97: 153-165, 1995.

5. Cornelius LA, Nehring LC, Roby JD, Parks WC and Welgus HG: Human dermal microvascular endothelial cells produce matrix metalloproteases in response to angiogenic factors and migration. J Invest Dermatol 105: 170-176, 1995.

6. Ingber DE, Madri JA and Folkman J: Endothelial growth factors and extracellular matrix regulate DNA synthesis through modulation of cell and nuclear expansion. In Vitro Cell Dev Biol 23: 387-394, 1987.

7. Brown JC and Timpl R: The collagen superfamily. Int Arch Allergy Immunol 107: 484-490, 1995.

8. Kalluri R: Basement membranes: structure, assembly and role in tumour angiogenesis. Nat Rev Cancer 3: 422-433, 2003.

9. Chang C and Werb Z: The many faces of metalloproteases: cell growth, invasion, angiogenesis and metastasis. Trends Cell Biol 11: 537-543, 2001

10. Xu J, Rodriguez D, Petitclerc E, et al: Proteolytic exposure of a cryptic site within collagen type IV is required for angiogenesis and tumor growth in vivo. J Cell Biol 154: 1069-1079, 2001.

11. Xu J, Rodriguez D, Kim JJ and Brooks PC: Generation of monoclonal antibodies to cryptic collagen sites by using subtractive immunization. Hybridoma 19: 375-385, 2000.
12. Brooks PC, Montgomery AM and Cheresh DA: Use of the 10-day old chicken embryo model for studying angiogenesis. Methods Mol Biol 129: 257-269, 1999.

13. Hoshida T, Sanamura M, Duda DG, et al: Gene therapy for pancreatic cancer using an adenovirus vector encoding soluble flt 1 vascular endothelial growth factor receptor. Pancreas 2: 111-121, 2002.

14. Hanahan D and Folkman J: Patterns and emerging mechanisms of the angiogenic switch during tumorigenesis. Cell 86: 353-364, 1996.

15. Folkman J: Tumor angiogenesis: therapeutic implication. $\mathrm{N}$ Engl J Med 285: 1182-1186, 1971

16. The kidney. In:. Robbins Pathologic Basis of Disease. 4th edition. Cotran RS, Kumar V and Robbins SL (eds). W.B. Saunders Company, Philadelphia, PA, pp1011-1082, 1989.

17. Holmes FA, Walters RS, Theriault RL, et al: Phase II trial of taxol, an active drug in the treatment of breast cancer. J Natl Cancer Inst 83: 1797-1805, 1991.

18. Weidner N, Semple JP, Welch WR and Folkman J: Tumor angiogenesis and metastasis - correlation in invasive breast carcinoma. N Engl J Med 324: 1-8, 1991

19. Longo R, Sarmiento R, Fanelli M, Capaccetti B, Gattuso D and Gasparinni G: Anti-angiogenic therapy: rationale, challenges and clinical studies. Angiogenesis 5: 237-256, 2002.

20. Ferrara N: VEGF as a therapeutic target in cancer. Oncology 69: $11-16,2005$

21. O'Reilly MS, Holmgren L, Shing Y, et al: Angiostatin: a novel angiogenesis inhibitor that mediates the suppression of metastases by a Lewis lung carcinoma. Cell 79: 315-328, 1994.

22. Brooks PC: Role of integrins in angiogenesis. Eur J Cancer 32A: 2423-2429, 1996

23. Seandel M, Noack-Kunnmann K, Zhu D, Aimes RT and Quigley JP: Growth factor-induced angiogenesis in vivo requires specific cleavage of fibrillar type I collagen. Blood 97: 2323-2332, 2001.

24. Senger DR and Perruzzi CA: Cell migration promoted by a potent GRGDS-containing thrombin-cleavage fragment of osteopontin. Biochim Biophys Acta 1314: 13-24, 1996.

25. Giannelli G, Falk-Marzillier J, Schiraldi O, Stetler-Stevenson WG and Quaranta V: Induction of cell migration by matrix metalloprotease-2 cleavage of laminin-5. Science 277: 225-228, 1997.

26. O'Reilly MS: Therapeutic strategies using inhibitors of angiogenesis. In: Methods and Molecular Biology. Vol. 223. El Deiry WS (ed). Humana Press, Totowa, NJ, pp599-633, 2003.

27. Teicher BA: A systems approach to cancer therapy. (Antioncogenics + Standard cytotoxics $>$ mechanism(s) of interaction). Cancer Metastasis Rev 5: 247-272, 1996.

28. Yardley DA: Gemcitabine plus paclitaxel in breast cancer. Semin Oncol 32 (suppl. 6): S14-S21, 2005.

29. Horwitz SB: Mechanisms of action of taxol. Trends Pharmacol Sci 13: 134-136, 1992. 